internationale qui a eu lieu à Berlin du 22 au 27 avril 1869, entre les délégués des puissances signataires dela Convention de Genève et des Sociétés de secours de presque tous les Etats de l'Europe. Tous les points les plus intéressants de la discussion sont indiqués, et les résolutions sont reproduites in extenso dans un appendice.

\title{
HYGIÈNE MILITAIRE
}

\section{Observations sur ses tendances actuelles ${ }^{1}$}

Il se manifeste actuellement de toute part une tendance qui commence à produire de bons résultats. Elle condnit à élargir le cercle d'action des médecins, que l'on ne considère plus comme devant n'agir qu'en présence des maladies déclarées, sans s'inquiéter des faits antérieurs.

On accentue maintenant, de plus en plus, par des paroles et des écrits, l'assertion émise par Pettenhofer, savoir: que la science médicale ne doit plus se contenter d'être l'esclave et la très-humble servante du public, accourant, lorsqu'on lui fait appel, pour remédier à un mal existant, puis, après s'être donné dans ce but plus ou moins de peine avec plus ou moins de succès, se retirant et recevant son salaire. On commence à soupconner qu'elle a le devoir de protéger les hommes et d'éloigner d'eux les dangers qui les menacent, même dans les cas où elle a en mains les moyens de guérir complétement les maladies qui viendraient à se déclarer.

Ce sont ces considérations qui ont déterminé la formation de la Section d'hygiène publique, à la quarante-unième assemblée des naturalistes et médecins de l'Allemagne à Francfort-sur-Mein ; et c'est par suite d'une résolution prise lors de la quarante-deuxième assemblée, tenue à Dresde en 1868, qu'a été fondée une feuille périodique traitant de l'hygiene publique. Il en a déjà paru trois livraisons sous le titre de: Revue trimestrielle allemande d'hygiène publique. ${ }^{2}$

Avant de passer à l'examen critique de leur contenu, nous dé-

1 Cet article de M. le Dr Roth est en majeure partie l'analyse d'un travail dont nous avons parlé dans notre premier Bulletin (page 20).

2 Voyez Bulletin No 1, page 19. 
sirons dire quelques mots du rapport intime qui existe, entre la tendance que nous venons de signaler et celle que la Convention internationale de Genève a inscrite sur sa bannière. On peut bien dire à la rigueur qu'elles ont un but commun, noble entre tous, savoir : la recherche et l'application des mesures les plus propres a préserver l'homme de maux qui proviennent, pour la plupart, de l'état social actuel. Cela est surtout vrai pour les calamités que prévoit la Convention de Genève, tandis que l'hygiène publique est appelée, en outre, à tenir compte de la lutte contre les forces de la nature. Nous ne méconnaissons nullement les difficultés inhérentes ả la première de ces catégories; nous les estimons même plus considérables que celles de la seconde; nous croyons néanmoins qu'en travaillant au développement de l'hygiène générale, au moyen d'une législation claire, ayant pleinement conscience de son but, nous obtiendrons souvent des résultats plus certains que l'on n'en pourrait compter, même après les efforts les plus énergiques tentés pour soulager les souffrances de milliers de blessés.

Mais il est un point sur lequel les travaux qui s'accomplissent dans ces deux domaines s'unissent étroitement; c'est qu'on déploie une égale sollicitude pour l'armée. Cette cuvre philanthropique des temps modernes doit emprunter une partie de ses moyens à l'hygiène, afin d'étendre préventivement sa main protectrice sur le soldat, et de lui épargner des maux qui peuvent l'atteindre, même sans qu'un seul coup de feu ait été tiré.

Les livraisons dont il s'agit traitent de toutes les branches de l'hygiène publique. Comme elles touchent à diverses professions, la Revue s'est assuré le concours d'hommes qui les représentent. C'est ainsi qu'elle compte au nombre de ses collaborateurs, - outre des médecins, - des architectes, des chimistes, des ingénieurs, des enployés de l'administration et des agronomes; elle offrira ainsi une grande variété d'articles.

Une circonstance que l'on peut considérer dès l'abord comme très-favorable à cette Revue, c'est que, jusqu'à présent, l'hygiène publique ne comptait aucun représentant dans la littérature périodique, et que les faits qui s'y rapportent se trouvaient généralement épars dans les journaux, notamment dans ceux consacrés à la médecine officielle, ainsi que dans ceux destinés aux ingénieurs.

Parmi les questions controversées, nous rencontrons au premier 
rang celle qui divise actuellement les écrivains, quant à l'hygiène publique, en deux grands partis, savoir : l'éloignement des matières fécales, ou la lutte entre la canalisation et l'enlèvement des déjections. La Revue trimestrielle allemande prend résolument parti, sous ce rapport, pour la canalisation, dont elle compte les principaux champions parmi ses collaborateurs ${ }^{1}$. La seconde livraison, notamment, contient à ce sujet des documents précieux, car on y expose d'une manière complète les travaux actuellement entrepris pour la canalisation de Dantzick. Les constructions actuelles des lazarets, en particulier des lazarets baraques, y sont de plus l'objet d'une description détaillée. Une revue très-complète de la législation anglaise, sur la salubrité publique, est d'autant mieux la bienvenue chez nous, qu'on l'ignore presque complétement et qu'en général nous n'en possédons point nous-mêmes. Un grand nombre de documents relatifs à presque toutes les branches de l'hygiène militaire, des dissertations critiques, etc., permettent d'embrasser d'un coup d'œil les progrès qui s'accomplissent dans cette science.

La Revue traite avec une sollicitude spéciale de l'hygiène militaire, à laquelle la littérature donne si peu d'attention comparativement à celle qu'elle accorde aux intérêts civils. Comme les progrès qui peuvent s'accomplir dans ce domaine touchent de très-près à l'œuvre de la Convention internationale de Genève, une rapide énumération des questions les plus importantes, parmi celles qui devront ètre soulevées plus tard à ce sujet, trouvera ici sa place naturelle.

La campagne de 1866 a ouvert les yeux du public, des savants, des militaires, sur les réformes à introduire dans l'armée, et en particulier sur l'organisation du service de santé.

Jusqu'à quel point cet objet mérite qu'on l'étudie, c'est ce qui ressort clairement des pertes que les maladies font éprouver aux armées. Ces pertes sont incomparablement plus fortes que celles causées par les blessures.

C'est ainsi que l'armée prussienne ne perdit, en 1866, que 4400 hommes par les armes à feu, tandis qu'il en mourut plus de 6000 à la suite de maladies.

${ }^{1}$ MM. Wiebe, conseiller privé aux travaux publics; Hobrecht, conseiller aux travaux publics; Latham, ingénieur. 
Les expériences faites dans toutes les guerres font donc, des moyens d'écarter ces sources de maux, une question du plus haut intérêt, pour tous ceux qui sont investis du commandement. La position que doit prendre le service de santé, pour se renáre compte autant que possible de toutes les influences nuisibles, ne peut être que celle-ci : il faut que les médecins militaires de tout grade soient tenus, sous peine d'assumer une lourde responsabilité, de faire rapport à ce sujet à leurs supérieurs respectifs, et cela sans qu'il soit besoin de les y inviter. Ces rapports, présentés par les membres du corps sanitaire, ne pourraient par contre être ignorés de l'officier qui commande, sans que son autorité en souffrît, à moins que cela n'eût lieu pour des motifs tout particuliers. De leur côté, les officiers seront d'autant plus accessibles à des représentations de cette nature, qu'ils seront mieux au courant de ces questions, ce qui leur fait un devoir d'acquérir sur cette matière une instruction convenable qui leur soit commune à tous. Cette instruction a été introduite dans l'armée de l'Allemagne du Nord, par des cours donnés à l'Académie militaire des officiers à Berlin. Il est de toute nécessité de fournir aussi sur ce sujet une instruction plus solide alux médecins militaires, et la meilleure manière d'obtenir ce résultat serait la formation d'écoles 'supérieures de médecine militaire, telles que l'Angleterre et la France en possèdent. Un moyen d'instruction, également excellent, serait de les exercer pendant un certain temps au maniement des armes. M. le médecin en chef Löffler a exprimé cette idée en termes éloquents, dans le discours qu'il a prononcé lors de la fête célébrée, le 2 août 1869, à l'Institut de médecine et de chirurgie de Frédéric-Guillaume à Berlin.

Il faut actuellement prendre pour modèle l'organisation de l'armée anglaise, pour laquelle, depuis la guerre de Crimée, ces questions ont été l'objet d'une étude approfondie. Sous ce rapport, il nous manque encore beaucoup de choses; ces lacunes nous sont d'ailleurs communes avec tous les pays, à l'exception de l'Angleterrè.

Quant aux questions spéciales qui peuvent amener des perfectionnements graduels dans l'hygiène militaire, il faut insister, en premier lieu, sur la nécessité d'améliorer considérablement la distribution de l'air dans les casernes. Ce progrès doit porter aussi bien 
sur l'augmentation du volume de l'air que sur la fréquence de son renouvellement.

Le gouvernement anglais seul, jusqu'à présent, a fait quelque chose dans ce sens, et pourtant la fièvre typhoïde et la phthisie pulmonaire étant la conséquence de l'air vicié, c'est justement le point sur lequel il importe le plus d'opérer une réforme.

Des ouvertures pour introduire l'air pur, des cheminées donnant issue à l'air vicié, combinées avec le chauffage des chambres, peuvent être recommandées comme de bons auxiliaires.

La fourniture de l'eau a également une importance énorme pour l'entretien de la santé. L'ingénieuse invention de l'américain Norton l'a beaucoup facilitée, même dans les circonstances difficiles, car elle permet d'emporter de l'eau partout, au moyen de cuves en fer aisément transportables. La qualité de l'eau, en tant que pouvant devenir une source de maladie, doit être l'objet d'une surveillance assidue.

Ces deux grands facteurs, l'air et l'eau, peuvent subir l'influence de la composition du sous-sol, laquelle dépend à son tour du procédé employé pour l'enlèvement des matières fécales.

Sous ce rapport, tout est actuellement encore dans le plus pitoyable état, et il est nécessaire, surtout dans les établissements militaires, oủ il y a accumulation d'hommes et d'animaux, qu'on y ait sérieusement égard. Des appareils d'écoulement, pourvus de tonneaux fermant hermétiquement, seront d'un bon usage pour les casernes. Les meilleurs procédés de désinfection restent sans effet, en l'absence d'un système régulier d'enlèvement des déjections.

Les camps offrent un excellent moyen de placer des troupes dans de bonnes conditions sanitaires, car, dès qu'ils sont établis, on peut procéder à un nettoyage à fond des casernes qui viennent d'ètre évacuées.

Les mesures suivantes doivent être considérées comme ayant une grande importance pour l'hygiène de l'armée dans les campements : choisir d'abord un emplacement aussi vaste que possible, puis faire en sorte que les tentes ne soient pas trop rapprochées, et les déplacer fréquemment. Toutes les déjections doivent être soigneusement enlevées, et brùlées si possible ; des appareils à ton- 
neaux peuvent également rendre de bons services, lorsqu'il s'agit d'enlever les matières fécales.

Les conditions sanitaires sont généralement moins satisfaisantes, dans les campements, avec les baraques qu'avec les tentes. A Châlons, il n'y a eu, en 7 ans, sous les tentes, que 1 homme malade sur 60, et dans les baraques, 1 sur 50. Il faut défendre de creuser la terre pour l'installation des tentes, vu que cela peut créer des causes d'insalubrité.

Les progrès accomplis dans l'art de conserver les aliments, ont beaucoup contribué à l'amélioration de l'hygiène alimentaire. L'extrait de viande, le lait conservé et le café au lait offrent le moyen d'emporter, en cas de détresse, le matériel de quelques repas au moins, ce qui est particulièrement important pour les lazarets de campagne.

Il y a quelques progrès à faire aussi dans l'art culinaire; l'école de cuisiniers fondée au camp d'Adershot peut servir de modèle aux autres armées. L'alimention introduite chez nous, dans les lazarets, peut à juste titre être qualifiée d'excellente.

L'habillement doit être commode et ne gêner ni la respiration ni la circulation du sang; à ce dernier point de vue il faut proscrire les cols hauts et raides. Il est de toute convenance de n'employer que des étoffes imperméables; quant à celles qui ne le sont pas, on peut parfaitement, leur donner cette propriété, en les brossant avec une solution d'alun mélangée à de l'amidon chaud, ainsi qu'en les imprégnant d'argile saturée de vinaigre, ce qui n'intercepte nullement le passage de l'air au travers des vêtements.

La manière de porter le havre-sac est également en progrès. Quoique jusqu'à présent le havre-sac prussien fùt celui qui approchait le plus de la perfection, la nouvelle manière anglaise de le porter est préférable, en ce qu'elle sépare les uns des autres le havre-sac, le manteau et les ustensiles de cuisine, et distribue ainsi le bagage sur toute la superficie du dos.

L'armée anglaise est en général la mieux habillée de toutes, chaque homme étant pourvu, outre son surtout imperméable et son uniforme, de vêtements fourrés pour une température septentrionale, et d'autres en coutil pour un climat tropical.

La propreté corporelle n'est pas suffisamment entretenue, l'ab- 
sence de conduites d'eau se faisant vivement sentir dans les casernes. Une cuve pour chaque centaine d'hommes, suivant l'usage anglais, est une proportion trop faible.

Il est de la plus haute importance, lorsque des épidémies viennent à éclater, de prendre à ce sujet les mesures convenables.

La plus fréquente des maladies internes passagères est la fière nerveuse ou typhus, qui se produit dans les armées par suite de l'encombrement et de l'infection du sol par les matières fécales. Dès qu'elle fait son apparition, il faut empêcher que les matières infectantes soient déposées ailleurs que dans les lieux spécialement destinés à cel usage, puis aussi faire camper sous la tente ou cantonner les soldats.

S'il s'agit du choléra, sur le caractère contagieux duquel il n'existe plus aucun doute, il est nécessaire, avant tout, d'éviter les mouvements de troupes qui pourraient propager la maladie; quant au reste, il faut aussi prendre bien soin d'enlever les excréments. Les conditions sanitaires individuelles des soldats exigent également une réglementation attentive de leur manière de vivre, dans tous ses détails.

Parmi les maladies chroniques, c'est la phthisie qui tient le premier rang. Comme le genre de vie du soldat peut influer sur son développement, on est pleinement autorisé à le libérer du service le plus tòt possible.

Quant à l'inflammation granuleuse des yeux, une propreté rigoureuse, aidée par une abondante fourniture d'eau, (chaque homme doit avoir sa cuvelte et son essuie-mains), et jointe à un air aussi pur que possible, est une condition indispensable pour préserver les soldats.

La syphilis exige avant tout que la prostitution soit réglée par des lois sévères. Un châtiment infligé aux soldats, seulement à cause de cette maladie, ne peut être qu'une mesure tout à fait insuffisante.

Parmi les maladies contractées pendant la marche, c'est l'insolation ou coup de soleil qui a le plus de gravité. Elle est occasionnée par une transpiration trop forte suivie de lassitude, par un habillement trop étroit qui gêne la respiration et la circulation du sang, par l'abus de l'eau-de-vie et l'air corrompu qu'on respire en mar- 
chant en colonnes serrées. Il faut, pour en préserver les soldats, leur permettre de boire de l'eau pendant la marche, ce qui met en même temps des bornes à l'abus de l'eau-de-vie; il faut leur permettre aussi d'ouvrir leurs cols, les faire marcher en rangs moins serrés et rendre leurs étapes plus fréquentes. Le café à l'eau et le thé sont les meilleures boissons que l'on puisse recommander aux soldats en marche.

Tous les faits concernant l'hygiène d'une armée doivent être annuellement consignés dans un tableau statistique clairement rédigé, et l'on aura ainsi posé le meilleur fondement sur lequel on puisse baser les réformes à entreprendre. On a fait le premier pas dans ce sens par la formation de la Section de médecine militaire, spécialement chargée de celte statistique.

L'œuvre internationale de secours aux blessés est à même de contribuer puissamment à ce que les vœux formulés dans cette notice soient exaucés, et cela, soit au moyen d'assistances de fait, en fournissant par exemple des conserves d'aliments, des appareils à filtrer l'eau, des étoffes imperméables, soit en agissant par son influence morale, continuant à élaborer des principes judicieux sur ces questions, et les faisant pénétrer parmi les soldats comme dans la population.

La haute portée de ces entreprises, qui répondent si bien aux aspirations humanitaires actuelles, justifie l'intérêt que leur ont voué les sociétés de secours.

$\mathrm{D}^{\mathrm{r}}$ Wilhelm Roтн. 